\title{
Annular Engine Development Status
}

\author{
Michael J. Patterson* \\ NASA Glenn Research Center, Cleveland, Ohio, 44135 \\ John E. Foster ${ }^{\dagger}$ \\ University of Michigan, Ann Arbor, MI, 48109 \\ Jason A. Young ${ }^{\star}$ and Mark W. Crofton ${ }^{\S}$ \\ The Aerospace Corporation, El Segundo, CA, 90245
}

\begin{abstract}
This publication documents the development status of the Annular-Geometry Ion Engine (AGI-Engine). The AGI-Engine is the design core for a new class of electric propulsion thrusters, referred to as the Next-Generation Electric Propulsion Thruster (NGEPT). The NGEPT is a single thruster concept, implementing an annular ring architecture, which potentially can be scaled and adapted for any power level at any specific impulse of interest. The AGI-Engine itself provides a pathway to substantial increases in thrust density $(\geq 9$ $\left.\mathrm{N} / \mathrm{m}^{2}\right)$, power density $\left(\geq 260 \mathrm{~kW} / \mathrm{m}^{2}\right)$ and input power (10's-100's of $\mathrm{kW}$ ) relative to conventional ion thrusters, at $<5000$ seconds specific impulse. Progress was demonstrated in the maturation of the concept with the design, fabrication, and testing of high-perveance flat annular geometry ion optics electrodes manufactured from pyrolytic graphite. The annular electrodes exhibit high beam collimation and high beam flatness while achieving a span-to-gap ratio of less than $1 / 2$ that of the NEXT ion optics with a beam area $\sim 97 \%$ of that of NEXT. Sufficient data were obtained to support the development of a full-scale highpower Annular Engine of $65 \mathrm{~cm}$ diameter with a potential input power capability of 21-62 kW over 2730-4670 seconds $I_{s p}$.
\end{abstract}

\begin{tabular}{|c|c|}
\hline & Nomenclature \\
\hline AGI-Engine & $=$ Annular-Geometry Ion-Engine \\
\hline DMH-Engine & $=$ Dual-Mode Hybrid-Engine \\
\hline EP & $=$ electric propulsion \\
\hline$F$ & $=$ thrust, $\mathrm{mN}$ \\
\hline GRC & $=$ Glenn Research Center \\
\hline HET & $=$ Hall-Effect Thruster \\
\hline$I_{b}$ & $=$ beam current, Amperes \\
\hline$I_{s p}$ & $=$ specific impulse, seconds \\
\hline MAGI-Engine & $=$ Multi-ring Annular-Geometry Ion-Engine \\
\hline NEXT & $=$ NASA's Evolutionary Xenon Thruster \\
\hline NGEPT & $=$ Next-Generation Electric Propulsion Thruster \\
\hline NSTAR & $=$ NASA Solar electric propulsion Technology Application Readiness \\
\hline PG & $=$ pyrolytic graphite \\
\hline$P_{i n-t}$ & $=$ thruster input power, $\mathrm{kW}$ (unless otherwise specified) \\
\hline SGR & $=$ span-to-gap ratio \\
\hline SOA & $=$ state of the art \\
\hline TAC & $=$ The Aerospace Corporation \\
\hline
\end{tabular}

\footnotetext{
* Sr. Technologist, Power and In-Space Propulsion Division, 21000 Brookpark Road/MS 301-3, AIAA Sr. Member

${ }^{\dagger}$ Associate Professor, Department of Nuclear Engineering and Radiological Sciences, 1906 Cooley Building 2355 Bonisteel Boulevard, AIAA Member

* Member of Technical Staff, Space Materials Laboratory, M2-341, P.O. Box 92957, Los Angeles, CA 90009

${ }^{\S}$ Sr. Scientist, Space Materials Laboratory, M2-341, P.O. Box 92957, Los Angeles, CA 90009, AIAA Sr. Member

American Institute of Aeronautics and Astronautics 


$\begin{array}{ll}\mathrm{TL} & =\text { throttle level } \\ V_{b p s} & =\text { beam power supply voltage, Volts } \\ \text { XIPS } & =\text { xenon ion propulsion system } \\ \eta_{t} & =\text { thruster efficiency }\end{array}$

\section{Introduction***}

THERE remain today many technical issues and barriers to the wider implementation of electric propulsion (EP). Some examples:

1. Conventional ion thruster designs have limited scalability to high power and limited thrust density. NASA investments have yielded major advances in ion propulsion technology: e.g. - the development of the NEXT ion propulsion system to Technology Readiness Level (TRL) 6 with a $6.9 \mathrm{~kW}$ thruster with a demonstrated total impulse of >33-million Newton-seconds; and the 2-khr demonstration of a TRL4 thruster operating at $21 \mathrm{~kW}$ (at 7650 seconds $I_{s p}$ ). ${ }^{1}$ Despite these advancements, and considerable agency resources applied toward its technology development over the last couple decades, ${ }^{2}$ the mission application of this technology by NASA and others are modest. This is because ion propulsion is generally 'relegated' to deep-space missions, and other energetic-mission applications which are not time-constrained but require very high propellant efficiency (e.g., auxiliary propulsion for geosynchronous station-keeping). This pigeonholing of ion propulsion occurs because conventional ion thruster designs have limited scalability in thrust density and power while simultaneously constraining the specific impulse to values of interest for less-energetic but more time-constrained missions, such as primary propulsion for Earth-orbital and cis-lunar applications. Similarly Hall-Effect Thrusters (HETs) are generally constrained to lower levels of specific impulse.

2. No single EP technology exists that is scalable in $I_{s p}(1500-5000$ seconds) at power levels of interest to the community. There are evolutionary paths following the status quo to develop a suite of different EP thruster technologies to meet NASA and other future mission needs. But this approach requires a multitude of technology development efforts, qualification tests, and life-tests with each requiring years of maturation, and each presenting unique spacecraft interaction challenges. So while the demand for a wide range of EP systems is greater than ever, the same time, the resources for thruster development and qualification are overly constrained to address the competing point design solutions for the numerous applications.

3. EP systems are costly to develop, qualify, and field. EP systems are generally designed and qualified as point designs targeted at a specific use for a specific spacecraft. When another user or application comes along substantial new effort must be performed. While the cost of a single thruster is typically in the low hundreds of thousands of dollars, qualification and integration of the EP system may cost tens of millions of dollars. Part of this issue lies in a lack of commonality in EP approaches among NASA, non-NASA government, and commercial users.

4. There is no present credible multi-100 kW EP approaches. This capability is important to support future human exploration architectures.

5. EP systems customers are risk averse. This hinders the implementation of new technology. While an EP system may have flown on numerous spacecraft, a scaled version to higher power is considered a new technology and categorized as high risk.

The Next-Generation Electric Propulsion Thruster (NGEPT) approach ${ }^{3}$ is proposed as a means to address these issues and barriers. The NGEPT concept:

1. Potentially enables scaling of ion thrusters to very high power (>100 kW) and high thrust densities at modest $I_{s p} ;$ and

2. Employs a reconfigurable design approach intended to capture the needs of all currently envisioned NASA, DoD and commercial mission applications, while expanding the application space of EP. Qualification of a reconfigurable NGEPT with expansive performance and life capabilities would eliminate the need to qualify multiple point designs, dramatically reducing the implementation cost of EP. The widespread use of NGEPT would increase manufacturing volume resulting in improved economy of scale and even larger cost savings.

\footnotetext{
** All concepts disclosed in the publication are Patent-Pending and are filed under both U.S. and International Patent Applications. Assignee: United State Government.
} 
The NGEPT is a novel, Glenn Research Center (GRC) patent-pending modular EP device based on a new arrangement of relatively mature and proven technologies. The design basis for the NGEPT is the AnnularGeometry Ion-Engine (AGI-Engine). The NGEPT concept is illustrated in Figure 1 in multiple potential variants. The development of the AGIEngine and its derivative design employed as a set of gridded-ion discharge chamber 'rings', in combination with other devices (e.g. - Hall accelerator 'rings') could yield reconfigurable thrusters for any power level and specific impulse of interest. The concept of developing and qualifying a set of nesting rings of annular ion engines, with and without centrally located HET channels, could result in a single thruster that could be qualified once over a wide range of Isp and power. The resulting product would be a reconfigurable thruster well suited for the entire range of applications and power levels from a few kilowatts to many 100's of kilowatts completed under a single development effort.

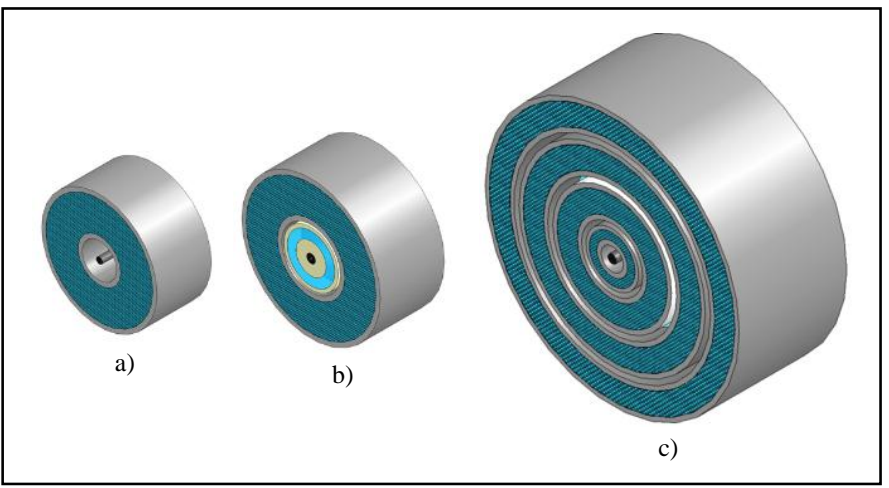

Figure 1. NGEPT Configurations: a) Annular Geometry Ion-Engine (AGI-Engine); b) Dual-Mode Hybrid-Engine (DMH-Engine), an AGI-Engine/HET Hybrid; and c) Multi-ring Annular Geometry-Ion Engine (MAGIEngine), from Ref. 3

The AGI-Engine itself has significant potential as a stand-alone thruster concept. These potentialities and technical descriptions are listed in Table 1, and include: very high thrust density; high power; improved efficiencies; enhanced life time; and improved packaging over state of the art (SOA) EP thrusters. ${ }^{4}$

Table 1. AGI-Engine Attributes and Technical Descriptions

\begin{tabular}{|c|l|}
\hline $\begin{array}{c}\text { Concept } \\
\text { Attribute }\end{array}$ & \multicolumn{1}{c|}{ Technical Description } \\
\hline $\begin{array}{c}\text { 3X Increase in } \\
\text { Thrust Density }\end{array}$ & $\begin{array}{l}\text { The annular discharge chamber increases the effective anode surface area for electron collection } \\
\text { as compared to a conventional cylindrically-shaped ion thruster of equivalent beam area. This } \\
\text { attribute allows operation at higher discharge currents and therefore high beam currents } \\
\text { yielding an increase in thrust density (up to 3X) and enabling near full-perveance capability of } \\
\text { the optics. An annular-geometry flat electrode may enable further electrostatic refinements } \\
\text { yielding higher-perveance designs with even higher thrust densities. }\end{array}$ \\
\hline $\begin{array}{c}\text { 10X Increase } \\
\text { in Power }\end{array}$ & $\begin{array}{l}\text { Instead of spanning a large discharge chamber diameter, an annular design enables a very large } \\
\text { beam area with relatively small electrode spans and relatively small span-to-gap ratios. This } \\
\text { mitigates the manufacturing, mechanical, and thermal challenges of large span spherically- } \\
\text { domed conventional ion optics, typically limited to about 600:1 span-to-gap. }\end{array}$ \\
\hline $\begin{array}{c}\text { Improved } \\
\text { Efficiencies }\end{array}$ & $\begin{array}{l}\text { Annular-geometry ion optics of small span may have sufficiently-high natural frequency to } \\
\text { implement flat electrodes. Flat electrodes will inherently yield improved efficiencies by } \\
\text { eliminating off-axis beam vectoring associated with spherically-domed ion optics electrodes } \\
\text { used on cylindrical thrusters; } ~ 3-4 \text { percentage point increase in efficiency across the } I_{s p} \text { range. }\end{array}$ \\
\hline $\begin{array}{c}\text { 10X Increase in } \\
\text { Life }\end{array}$ & $\begin{array}{l}\text { Because of the relatively-simple physical design of the electrodes for an AGI-Engine, the } \\
\text { present manufacturing limitations with carbon are circumvented, thereby allowing the practical } \\
\text { implementation of carbon and the life time enhancements of this material. }\end{array}$ \\
\hline $\begin{array}{c}\text { Improved } \\
\text { Packaging }\end{array}$ & $\begin{array}{l}\text { The annular discharge chamber design allows central mounting of the neutralizer within the } \\
\text { annulus, and the potential to incorporate multiple annular discharges packaged into concentric } \\
\text { rings, thereby reducing the outer profile of the engine. This would also allow for a shared } \\
\text { gimbal platform. }\end{array}$ \\
\hline
\end{tabular}

Under a Phase 1 effort a sub-scale proof-of-concept breadboard AGI-Engine discharge was designed, fabricated and tested for purposes of evaluating the efficacy of the concept, the results of which were reported previously. ${ }^{3,5}$ The breadboard engine was built from a combination of components originally manufactured for a NEXT $40 \mathrm{~cm}$ ion thruster and those specifically manufactured for the annular engine design. These tests were successful in demonstrating stable annular discharge operation, and uniform annular plasma densities, using a singular discharge hollow cathode with an optimized magnetic circuit. Estimated discharge losses under conditions simulating beam 
extraction were low and consistent with those values required to yield high overall engine efficiency. The breadboard AGI-Engine was subsequently modified for operation with beam extraction, including integration of NEXT ion optics modified to simulate an annular configuration by covering the central $8 \mathrm{~cm}$ diameter portion of the electrodes. Tests with beam extraction over a range of throttle levels developed for the NEXT thruster verified both low discharge losses ( $200 \mathrm{~W} / \mathrm{A})^{3}$, and a highly-uniform beam current density profile (0.85-0.95 beam flatness). ${ }^{5}$ Optics and overall engine performance were consistent with expectations.

Based on the positive results achieved with the sub-scale breadboard AGI-Engine further evaluation of the concept was initiated. Table 2 lists the sub-scale AGI-Engine and full-scale AGI-Engine development tasks and status required for concept maturation. These activities include: design, manufacturing, test and evaluations of subscale flat carbon annular ion optics; and design of a larger high-power (1-2 Newton class) AGI-Engine to assess concept scalability. These topics are discussed in this publication.

Table 2. AGI-Engine Tasks and Status

\begin{tabular}{|l|c|}
\hline \multicolumn{1}{|c|}{ Phase 1-Sub-Scale Proof-of-Concept Annular Engine Tasks } & Status \\
\hline 1. Demonstrate Stable Annular Discharge Operation & Demonstrated \\
\hline 2. Demonstrate Uniform Annular Plasma Densities & Demonstrated \\
\hline 3. Demonstrate Low (<400 W/A) Discharge Losses & Demonstrated \\
\hline 4. Demonstrate Uniform Beam Current Density & Demonstrated \\
\hline 5. Demonstrate Feasibility of Annular Ion Optics at Sub-Scale & To Be Demonstrated \\
\hline 6. Demonstrate Carbon-Based Annular Ion Optics & To Be Demonstrated \\
\hline 7. Demonstrate Feasibility of Flat Annular Ion Optics & To Be Demonstrated \\
\hline \multicolumn{1}{|c|}{ Phase 2 - Full-Scale High-Power Annular Engine Tasks } & Status \\
\hline 1. Design and Fabrication & In Process \\
\hline 2. Demonstrate Scalability of Annular Discharge-and-Ion Optics & To Be Demonstrated \\
\hline 3. Demonstrate Higher Supportable Discharge Currents than SOA & To Be Demonstrated \\
\hline 4. Demonstrate Higher Beam Current Densities than SOA & To Be Demonstrated \\
\hline
\end{tabular}

\section{Sub-Scale Annular Engine Development - Carbon-based Annular Ion Optics}

This section discusses the sub-scale breadboard AGI-Engine status; in particular development of carbon-based flat annular ion optics. Fabrication and preliminary test results are presented.

\section{A. Design and Fabrication}

Application of carbon electrodes for ion thrusters is advantageous due to its nearly order-of-magnitude improvement in life capability associated with its reduced sputter-yield relative to conventional refractory metals. The implementation of carbon in both carbon-composite and pyrolytic graphite (PG) forms for ion thruster electrodes have to date been inhibited by manufacturing limitations. The implementation of PG has met with some success in GRC's development of both $8 \mathrm{~cm}$ diameter (flat electrode) and $30 \mathrm{~cm}$ diameter (spherically-domed electrode) high-perveance ion optics. ${ }^{6,7}$ However its fabrication in a $40 \mathrm{~cm}$ diameter spherically-domed geometry (for compatibility with the NEXT ion thruster) - requiring the use of a 5-axis milling machine - has been challenging. The high open-area-fraction to achieve high-perveance makes the fabrication of spherically-domed electrodes an extremely-lengthy and costly process subject to error and mechanical failure.

In contrast, the machining of flat-panel PG should be rapid and relatively-low cost. Annular-geometry ion optics of relatively small span should have a sufficiently-high first mode natural frequency to allow for the application of flat electrodes. Because of the relatively simple physical design of the electrodes, the manufacturing limitations associated with carbon for the electrodes on conventional ion thrusters are then circumvented - thereby potentially enabling the practical implementation of carbon and hence the life time enhancements of this material.

A design for flat sub-scale PG annular ion optics electrodes was completed. The design employed a flat-panel 2electrode configuration with annular panel dimensions of $46 \mathrm{~cm}$ outside diameter and $5.1 \mathrm{~cm}$ inside diameter, compatible with mechanical integration onto the breadboard discharge chamber. The dimensions of the active area - the annulus within which ion beam apertures would be placed - was $36.4 \mathrm{~cm}$ outside diameter and $7.6 \mathrm{~cm}$ inside diameter, matching the internal dimensions of the breadboard discharge chamber plasma production region. The active area dimensions yield a beam area of approximately $995 \mathrm{~cm}^{2}$; about 0.97 that of the NEXT ion thruster optics 
beam area. Other critical dimensions associated with the electrodes and aperture patterns (e.g., sub-millimeter screen and accelerator electrode thicknesses, sub-millimeter screen-to-accelerator interelectrode gap, implemented as a high open-area-fraction hexagonal array of circular apertures) were chosen to match the high-perveance design of the conventional refractory metal NEXT ion thruster optics.

Manufacturing of the electrodes was conducted at GRC using previously-procured PG raw stock in the form of thick $(7.6 \mathrm{~mm})$ panels. Machining was required to reduce the material thickness from its initial value to two (different) final sub-millimeter thickness sheets, one each for the screen and accelerator electrodes. Machining was identified as a less-costly approach than either procuring new panels or slicing the existing panels to near-final thickness. However this did require a new manufacturing process; a vacuum hold-down fixture was developed to maintain the PG material in a fixed plane during successive machining passes. This technique successfully demonstrated machining of PG sheet to final thicknesses of $<0.13 \mathrm{~mm}$. This demonstration of the ability to machine PG to very fine and uniform thickness (factors below conventional electrode thicknesses) over large area may be of value in the future implementation of alternative very-high perveance electrode geometries.

Post-machining, each annular-shaped electrode blank underwent a separate milling process to machine $\sim 23,000$ matched through-holes using a 3axis CNC mill to create a precisely-controlled hexagonal array pattern required for the electrostatic design. The finished pyrolytic graphite electrodes achieved all the required dimensional tolerances, and are shown in Figure 2. A manufacturing yield of $100 \%$, with zero aperture defects, was demonstrated.

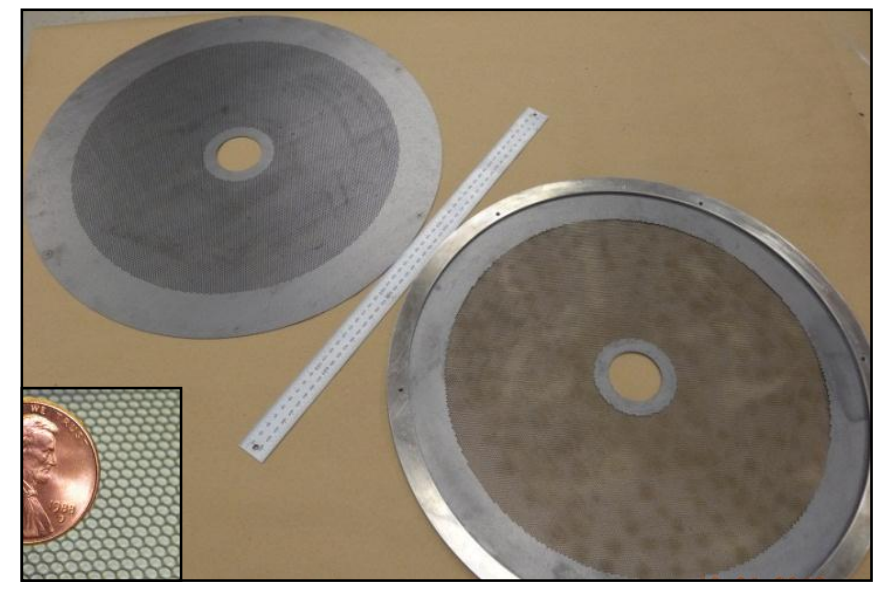

Figure 2. Sub-Scale Flat PG Annular Electrodes: Accelerator Electrode Upper-Left; Higher Open-AreaFraction Screen Electrode Lower Right. Half-Meter Ruler Show for Scale (Al ring shown on Screen Electrode is assembly-aid only). Screen Apertures are shown in the Left Insert Photo.

\section{B. Electrostatic Bench Tests}

Once the PG electrodes were manufactured they were aligned and assembled into a 2-electrode optics configuration. A very simple laboratory-version mounting scheme was employed using a titanium mounting ring (built for $40 \mathrm{~cm}$ NEXT ion optics) and sheet mica of approximately $0.64 \mathrm{~mm}$ thickness to electrically isolate the electrodes, with the entire assembly held in compression with paper binders (reference Fig. 3). This method of electrode mounting has been successfully employed previously for both bench top and active beamextraction tests of operational ion engines, and is a quick method of verifying electrostatic functionality of new designs before committing the resources

toward the manufacturing of a thermally- and structurally-compliant flight-like mounting assembly. In this configuration the ion optics have

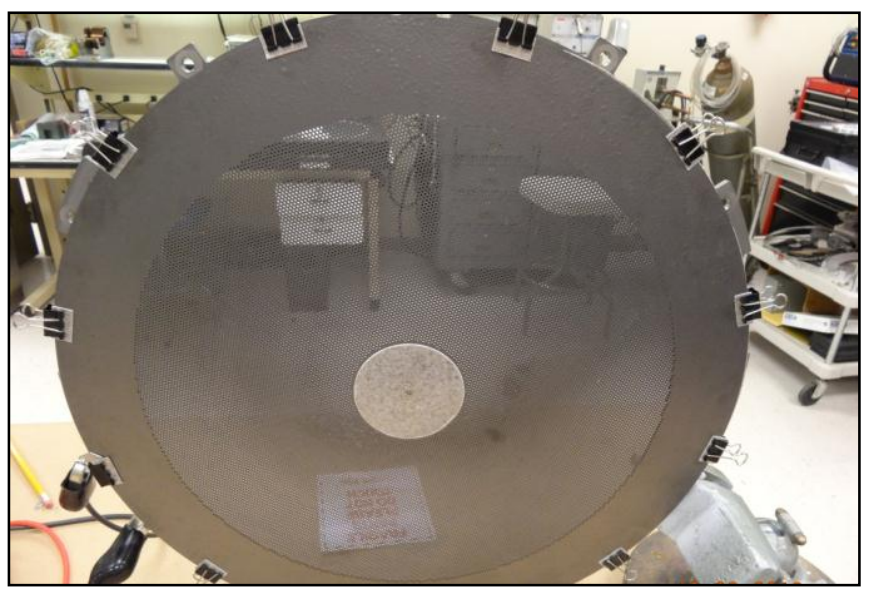

Figure 3. Electrostatic Bench Testing of Flat Annular PG Electrodes in an Ion Optics Configuration an effective span-to-gap ratio (SGR) of about 227; less than half that of the NEXT ion optics.

The objectives of the electrostatic tests were to assess the magnitude of the electrostatic deflection of the flat annular PG electrodes, and document the achievable SGR as a function of applied voltage and unsupported span. This was accomplished by varying the applied voltage across the electrodes, and changing the unsupported span by varying the inside diameter of the interstitial mica placed between the electrodes. These data provide critical 
parametric information essential to determine the design trade-space available for flat PG ion optics: electrode thickness; unsupported span of the annulus; inter-electrode gap; and applied voltage.

The preliminary results indicated that unsupported spans of at least $14.4 \mathrm{~cm}$ - the total span of the beam extraction area for the manufactured PG electrodes - are feasible in a flat pattern using the baseline screen electrode thickness at electric field strengths up to $2400 \mathrm{~V} / \mathrm{mm}$ without incurring significant electrostatic deflection. The implications of these results are that it should be feasible to develop high perveance ion optics in a flat geometry pattern, and hence reap the benefits of a flat design; namely, improved performance and reduced fabrication cost.

\section{Preliminary Beam Extraction Tests}

Preliminary beam extraction tests of the flat PG electrode assembly were conducted with the breadboard AGI-Engine discharge. The breadboard AGIEngine discharge has an $8 \mathrm{~cm}$ inside diameter and $42 \mathrm{~cm}$ outside diameter. A singular hollow cathode was installed at a radial position corresponding to the center of the annulus. The effective anode surface area for the breadboard AGIEngine discharge was $\sim 1.5 \mathrm{X}$ that of the NEXT thruster. A more detailed description of the AGI-Engine discharge can be found in Ref. 3. The assembled configuration is shown in Figure 4.

The objectives of the beam extraction tests were to: (a) demonstrate stable beam extraction and operation with flat PG electrodes; (b) obtain preliminary estimates of discharge losses (discharge electrical efficiency) and overall thruster performance; and (c) obtain preliminary measurements of beam characteristics (beam current density profile and beam divergence).

A laboratory propellant feed system and commercial power supplies were used to operate the AGI-Engine. Testing was executed at The Aerospace Corporation's (TAC's) Electric Propulsion 2 (EP2) space simulation facility in El Segundo, CA. EP2 facility has dimensions of approximately 2.4 meters diameter

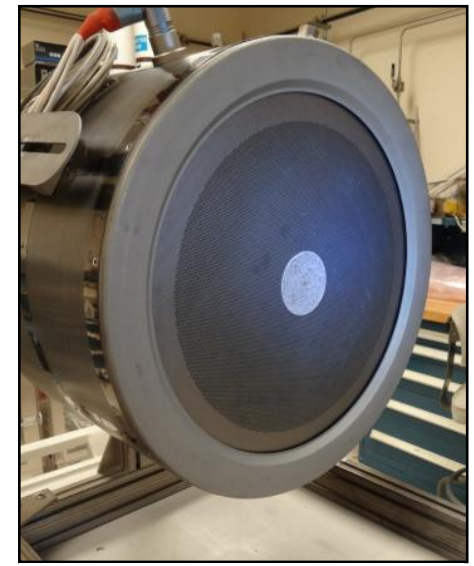

Figure 4. Sub-Scale AGIEngine with Flat PG Electrodes by 9.4 meters length, with a pumping speed of $\sim 200$ kiloliters per second on Xe. Performance of the engine was calculated by standard convention using a methodology documented in reference $8 . \dagger$

These first series of tests were truncated because of an implementation error with the laboratory-version mounting arrangement, reducing the data acquired. Six matched-set of through-holes were included in both electrodes at large radius, to be used as alignment guides during assembly. The radial location of these holes was slightly inboard of the inside radius of the titanium mounting ring, thus exposing them to discharge chamber plasma. Although sheet mica was placed between the electrodes at these locations, the exposed mica within the holes was subject to ion bombardment from ions accelerated from the discharge plasma, causing degradation of its insulation properties resulting in relatively rapid development of a short across the optics at these locations. A simple fix to eliminate this shorting in future tests is to apply a metal shim over the alignment apertures on the upstream surface of the screen electrode.

Despite this issue the flat PG electrodes were operated with beam extraction for several 10's of hours in total, at electric fields up to about 2200 $\mathrm{V} / \mathrm{mm}$, over a range of throttle levels (TLs) associated with the NEXT thruster throttle table., These TLs (identified in the yellow highlighted cell of Table 3) included: TL05, TL06, TL07, TL08, and TL09, with corresponding beam power supply voltages $\left(V_{b p s}\right)$ ranging from $679-1179$ volts, all at 1.20 Amperes beam current $\left(I_{b}\right)$; and TL14 at 1179
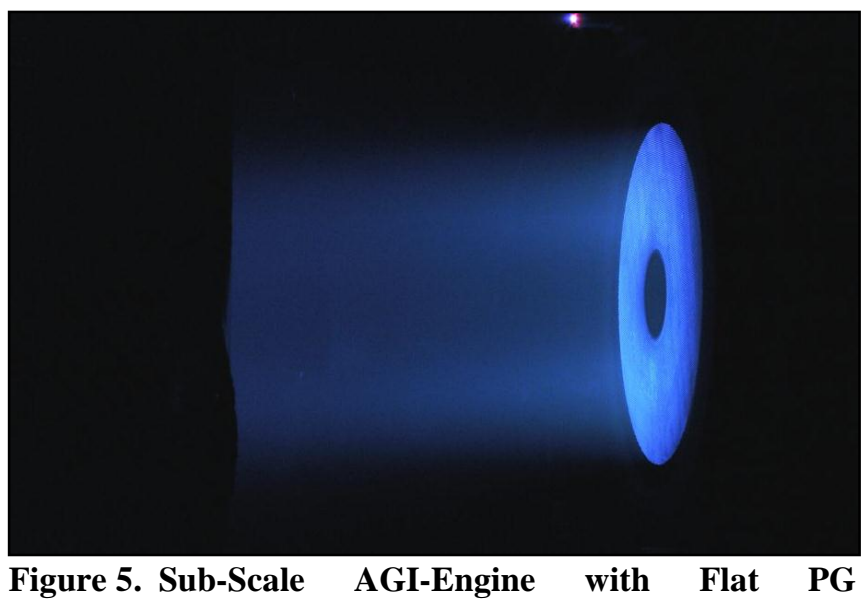
Electrodes Operating under Beam-Extraction Testing at Aerospace Corporation; Neutralizer at 12-O'clock Position

\footnotetext{
${ }^{1 \dagger}$ The performance equations account for items including neutralizer propellant flow and power consumed, as well as a correction to discharge chamber propellant efficiency for re-ingested propellant flow due to elevated facility pressure. Thrust loss correction factors include corrections for estimated beam charge-state and measured beam divergence due to off-axis vectoring.
} 
volts $V_{b p s}$ and 1.60 Amperes $I_{b}$, corresponding to a maximum thruster input power $\left(P_{i n-t}\right)$ of about $2.4 \mathrm{~kW}$. The NEXT TL's consist of 40 discrete operating points which are defined by a unique combination of beam current and beam power supply voltage - or equivalently input power and specific impulse, as illustrated by the highlighted cells in Table 3.

Table 3. NEXT Thruster Standard Throttle Levels (Green and Yellow Highlighted Cells)

\begin{tabular}{|c|c|c|c|c|c|c|c|c|c|c|c|c|}
\hline \multicolumn{13}{|c|}{$V_{b p s}, \mathrm{~V}$} \\
\hline$I_{b}, \mathrm{~A}$ & 1800 & 1567 & 1396 & 1179 & 1021 & 936 & 850 & 679 & 650 & 400 & 300 & 275 \\
\hline 3.52 & TL40 & TL39 & TL38 & TL37 & & & & & & & & \\
\hline 3.10 & TL36 & TL35 & TL34 & TL33 & & & & & & & & \\
\hline 2.70 & TL32 & TL31 & TL30 & TL29 & TL28 & & & & & & & \\
\hline 2.35 & TL27 & TL26 & TL25 & TL24 & TL23 & & & & & & & \\
\hline 2.00 & TL22 & TL21 & TL20 & TL19 & TL18 & & & & & & & \\
\hline 1.60 & TL17 & TL16 & TL15 & TL14 & TL13 & & & & & & & \\
\hline 1.20 & TL12 & TL11 & TL10 & TL09 & TL08 & TL07 & TL06 & TL05 & TL04 & TL03 & TL02 & \\
\hline 1.00 & & & & & & & & & & & & TL01 \\
\hline
\end{tabular}

Figure 5 is a photo of the AGI-Engine operating with the flat PG electrodes operating under beam extraction; note highly-collimated ion beam propagating from right-to-left. Other than the aforementioned electrode shorting (occurring outside the active beam area) the PG electrodes performed well, with a low breakdown (arcing) frequency typical of that experienced with conventional metal electrodes. There were no excessive arcs in the active beam area, or shorting of the electrodes due to excessive electrostatic deflection.

Although there was insufficient operating duration to quantify the optics perveance, a slightly-reduced perveance from the very-high level experienced with the NEXT ion optics was noted from observation of the accelerator electrode impingement current with TL. This is likely associated with the reduced beam area of the annular electrodes, and possibly larger interelectrode-gap at operating-temperature, as compared to the NEXT optics metal electrodes.

Discharge losses (the discharge power required to produce an Ampere of beam ion current), and overall thruster performance, were documented for the aforementioned TLs. Figure 6 plots the measured discharge losses for the AGIEngine operating with the flat annular PG electrodes versus TL. Also plotted are the equivalent data measured when this same annular discharge chamber was tested

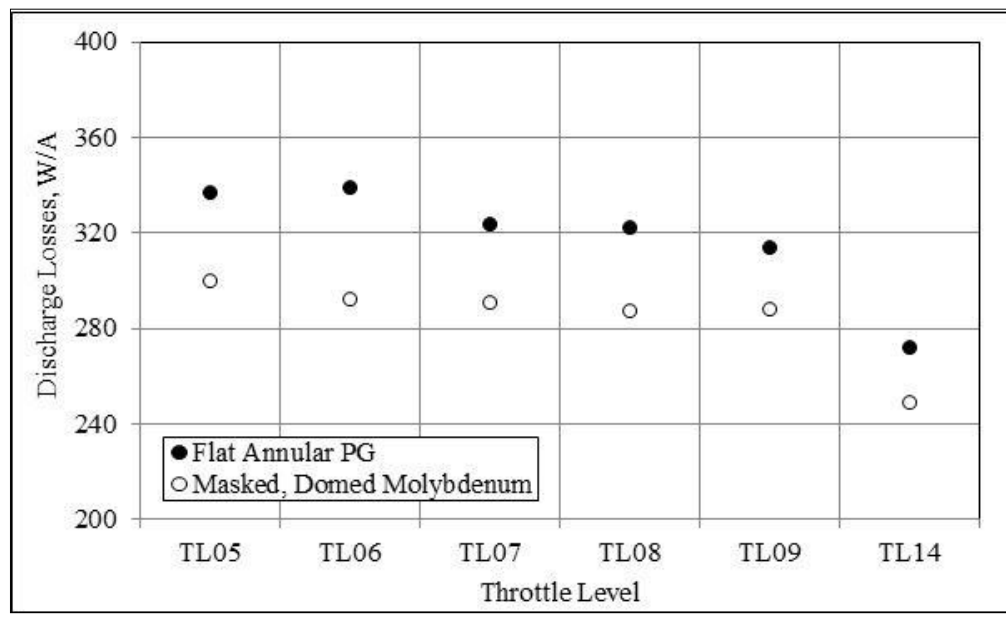

Figure 6. Comparison of Sub-Scale AGI-Engine Discharge Losses for Two Electrode Geometries at Various TLs

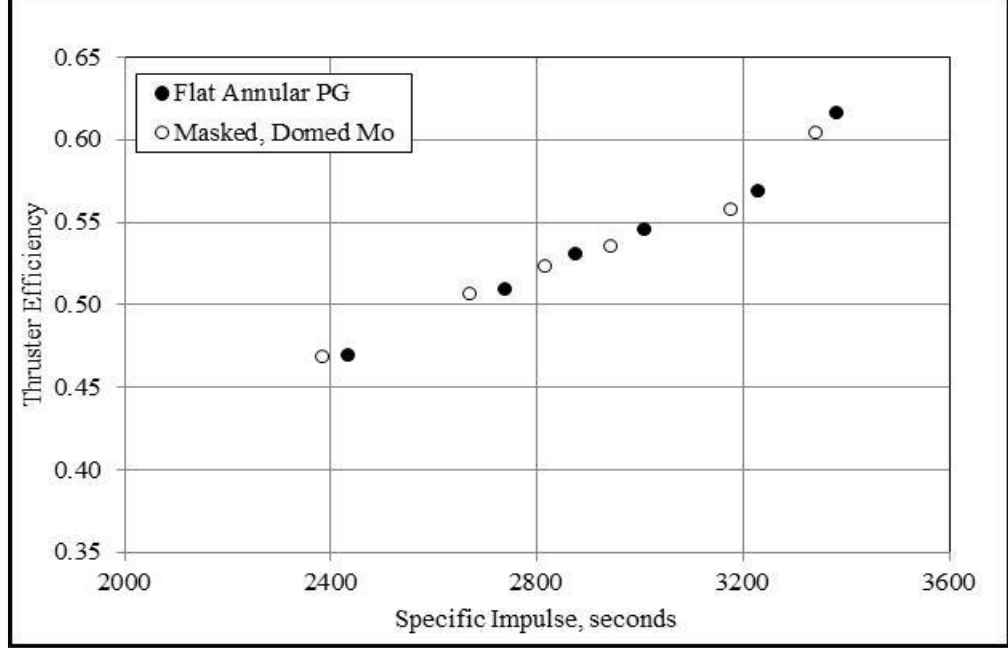

Figure 7. Efficiency vs. Specific Impulse for Sub-Scale AGI-Engine with Two Electrode Geometries 
with NEXT ion optics masked into an annular pattern, reported previously. ${ }^{3}$ As indicated the discharge losses experienced with the flat annular PG electrodes is higher, by about an average of $\sim 11 \%$, over the range of TLs investigated. The electrode patterns exposed to the discharge of both sets of ion optics are identical (including screen electrode) with the one exception that the molybdenum electrodes have a spherically-domed convex shape and the PG electrodes are flat. One explanation for the difference in discharge losses is that installation of the flat PG electrodes resulted in a reduction in discharge chamber ion production volume (of about $8 \%$ ) as compared to the domed molybdenum electrodes, simply because of the loss of volume upstream of the dome. Effectively, the discharge chamber length was decreased when switching to the flat annular PG electrodes. Also it is likely that the interelectrode gap for the molybdenum electrodes is slightly smaller than that of the PG electrodes while under thermal load, resulting in improved ion transparency and hence reduced discharge losses. In any event, the magnitudes of the discharge losses are reasonably low operating with the PG electrodes; below the 400 W/A target. The trends in discharge losses with TL are consistent with expectations and prior data; decreasing both with increasing total voltage due to improved ion transparency, and decreasing with increasing $I_{b}$.

Overall AGI-Engine performance at these TLs with the flat PG electrodes is comparable to that previously documented with Mo electrodes on this discharge chamber, ${ }^{3}$ as shown in Figure 7, a plot of thruster efficiency $\left(\eta_{t}\right)$ vs. $I_{s p}$. The engine performance analysis includes a correction factor for beam divergence established from plume measurements over a range of axial distances. The thrust loss divergence correction factor for the flat PG electrodes was determined to be 0.997 ; significantly higher than that measured for the domed NEXT optics of $\sim 0.96-0.97$. $^{9}$

Beam current density profiles for the flat PG electrodes were obtained at all TLs characterized. A typical current density profile scan is shown in Figure 8 for TL08 (1.20 Amperes $I_{b}$ and $1021 \mathrm{~V}$ $V_{b p s}$ ) obtained at an axial distance of 25 $\mathrm{mm}$. Also plotted is a current density profile reported previously for the masked, domed Mo electrodes at the same TL on the AGI-Engine. ${ }^{5}$ The beam current density profiles and (high) flatness parameters with the flat PG electrodes compare favorably to that documented on the discharge chamber with the masked, domed Mo electrodes. The background flux at the center is larger for the flat PG electrodes but the tail at high angle are narrower, consistent with a more-

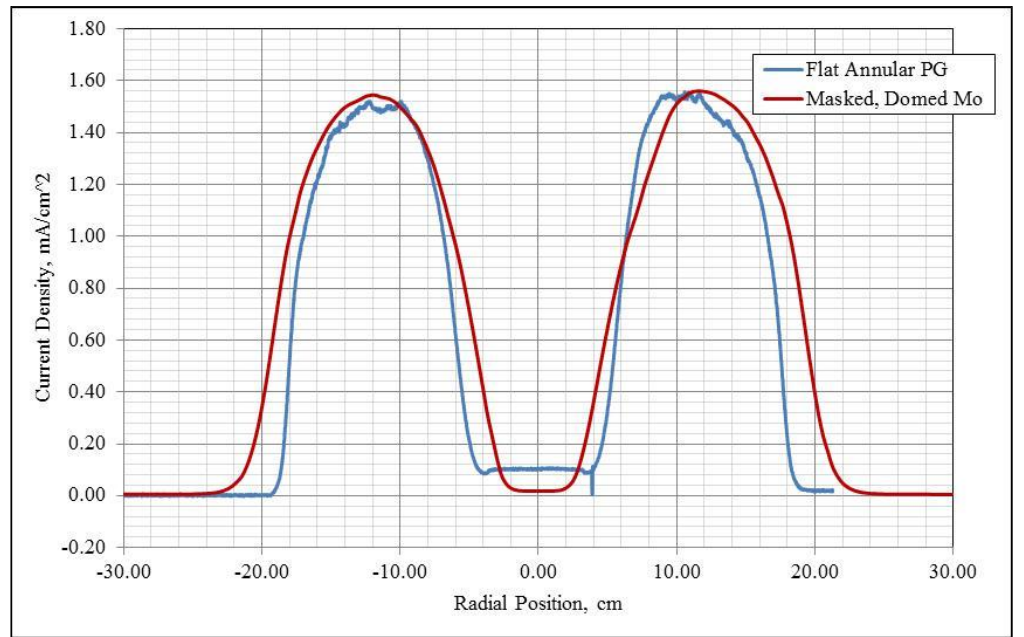

Figure 8. Current Density Profiles for Sub-Scale AGI-Engine with Two Electrode Geometries at TL08 collimated ion beam.

In summary these tests successfully demonstrated the remaining tasks identified in Table 2 for the sub-scale proof-of-concept Annular Engine. While additional testing of the sub-scale AGI-Engine is warranted to completely document the electrostatic behavior of the PG electrodes, sufficient data were obtained to support the design, assembly, and demonstration testing of a full-scale high-power Annular Engine:

- Large-diameter flat annular geometry electrodes of high-perveance design were successfully manufactured from pyrolytic graphite;

- The PG electrodes were integrated with the annular discharge chamber, and by stable operation at multiple NEXT thruster TL's demonstrated relatively high perveance, with very-high collimation and high beam flatness;

- A span-to-gap ratio (SGR) of less than $1 / 2$ that of the NEXT ion optics was demonstrated at $97 \%$ of the NEXT beam area, at electric fields up to about $2200 \mathrm{~V} / \mathrm{mm}$.

\section{Concept Studies}

The AGI-Engine concept has the potential to dramatically increase the operational envelope of ion thrusters, to high thrust density and very high power at moderate (sub-5000 second) specific impulse. The following section discusses some issues and practical considerations with respect to scaling up the AGI-Engine concept from the subscale breadboard to larger engines, and considerations relative to development and practical implementation. 
A. Scalability and Scaling Relationships

1. Maximum Annulus Electrode Span and Electric Field Strength

Increases in both unsupported span $(>14.4 \mathrm{~cm})$ and in electric field strength for flat PG electrodes beyond that demonstrated with the sub-scale flat PG electrodes are possible. One means of doing so would be to increase the screen electrode thickness and hence its stiffness. To this end a second PG screen electrode was fabricated, identical in all aspects to the first screen electrode with the exception that it is $133 \%$ as thick, which will be the subject of future investigation and reporting. Increases in screen electrode thickness to improve stiffness will however reduce the perveance. Figure 9 illustrates the change in normalized perveance with change in (normalized) screen electrode thickness; calculated using a non-planar sheath model approximation for a 2-electrode

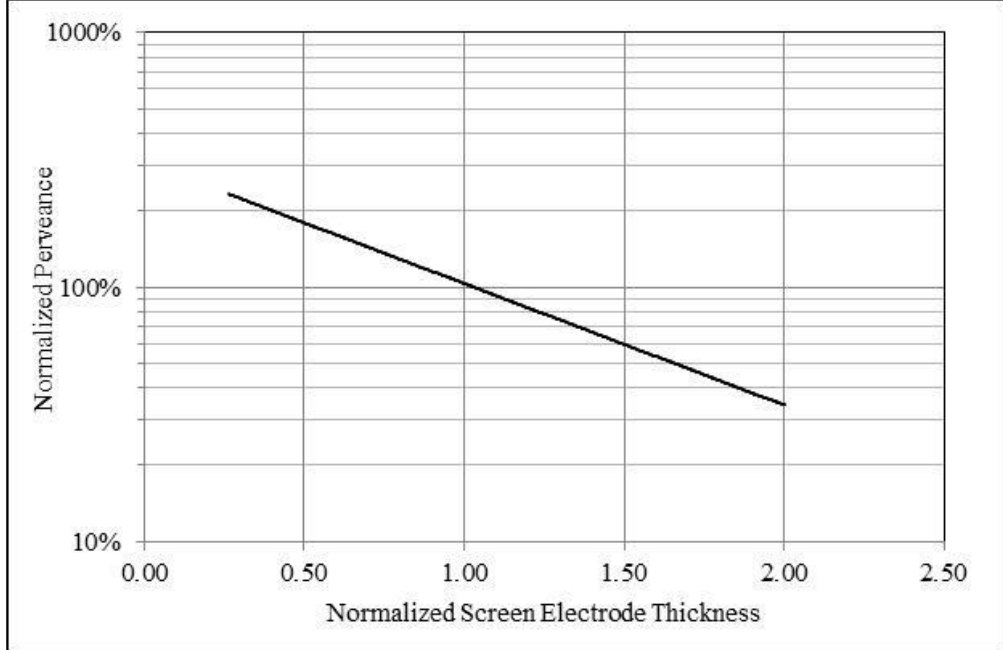

Figure 9. Normalized Perveance as a Function of (Normalized) Screen Electrode Thickness

geometry. ${ }^{10}$ A $133 \%$ increase in screen electrode thickness is estimated to yield a reduction in about $30 \%$ in normalized perveance, but with about a $2.4 \mathrm{X}$ increase in stiffness.

The reduction in perveance associated with increased screen electrode thickness may be compensated for by reducing the interelectrode gap. Figure 10 illustrates the normalized interelectrode gap required to fully-recover the perveance as a function of (normalized) screen electrode thickness. As noted, modest adjustments in gap - e.g., a reduction to about 0.81 of the initial value - would fully-recover the lost perveance associated with a $133 \%$ increase in screen electrode thickness. Alternative approaches to recovering perveance, e.g., changing the aperture geometry from a straight-cylinder to a tapered-cylinder, should be straightforward to implement using the manufacturing processes required for machining of apertures in PG electrodes. Of course maximum unsupported span and electric field strength can be directly

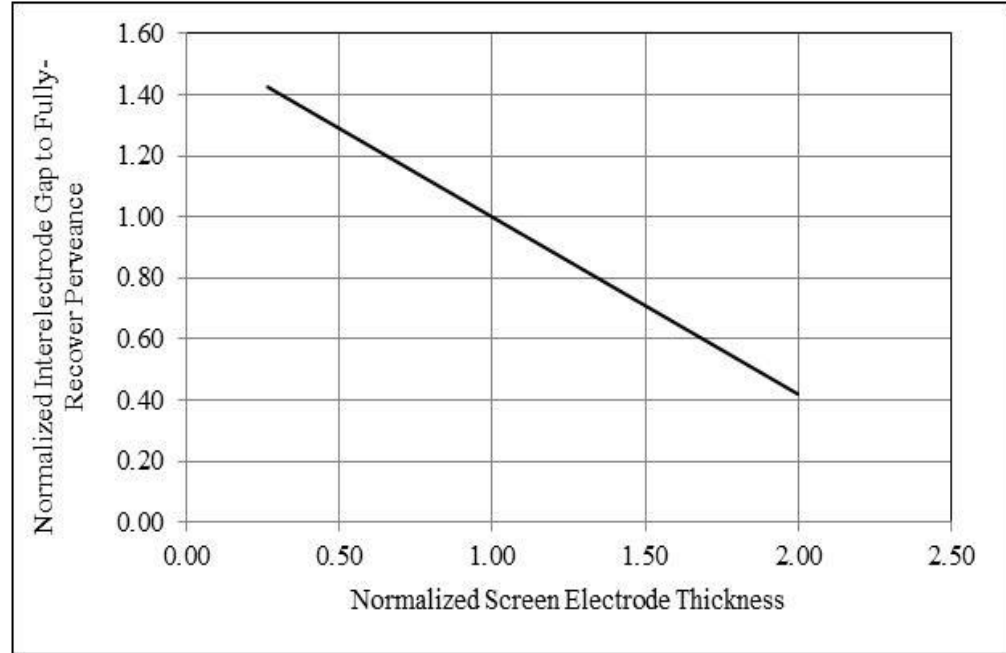

Figure 10. Normalized Interelectrode Gap to Fully-Recover Perveance as a Function of (Normalized) Screen Electrode Thickness

traded off. Further parametric investigations are warranted to fully examine the design trade-space available for flat PG ion optics.

\section{Maximum Beam Diameter}

Annular-geometry electrodes may allow for scaling of ion thrusters to very high power by enabling the fabrication of large area high-perveance (sub-millimeter thick electrodes with sub-millimeter interelectrode gap) ion optics, by providing a design approach which limits the required electrode span and SGR. A simple illustration of this is shown in Figure 11, a plot of the required SGR as a function of beam diameter necessary to replicate the high perveance design of conventional (spherically-domed circular electrodes) ion optics employed on SOA on thrusters of $\leq 0.40 \mathrm{~m}$ beam diameter. As indicated to manufacture high-perveance ion optics of conventional design greater than about $0.75 \mathrm{~m}$ diameter requires an SGR of $>1000$, whereas the practical limit (from a manufacturing and assembly tolerance perspective) is in the range of $650-700 \mathrm{SGR}$, which constrains the maximum diameter to $\approx 0.45$ 
m. Alternatively with an annular electrode approach large beam diameters can be achieved at relatively low SGR; e.g., in the case demonstrated here $36.4 \mathrm{~cm}$ beam diameter at an SGR of $\sim 227$, which is less than $1 / 2$ the SGR using a conventional electrode approach.

However there are manufacturing limitations to the fabrication size of PG, which is the leading candidate electrode material. Present manufacturing issues, associated with furnace size, limit individual PG panel sizes to about $66 \mathrm{~cm}$ diameter. Even with this constraint this still represents a substantial increase in beam area, as compared to that demonstrated with conventional ion thrusters. For example, a $65 \mathrm{~cm}$ beam diameter AGI-Engine, with a maximum span of $14.4 \mathrm{~cm}$, would yield a beam area of approximately $2290 \mathrm{~cm}^{2}$ which is $\sim 2.25 \mathrm{X}$ that of the NEXT thruster beam area. If this $65 \mathrm{~cm}$ AGI-Engine were then simply operated at the same peak power density as that previously demonstrated with the NEXT thruster $^{8}$ it would have an

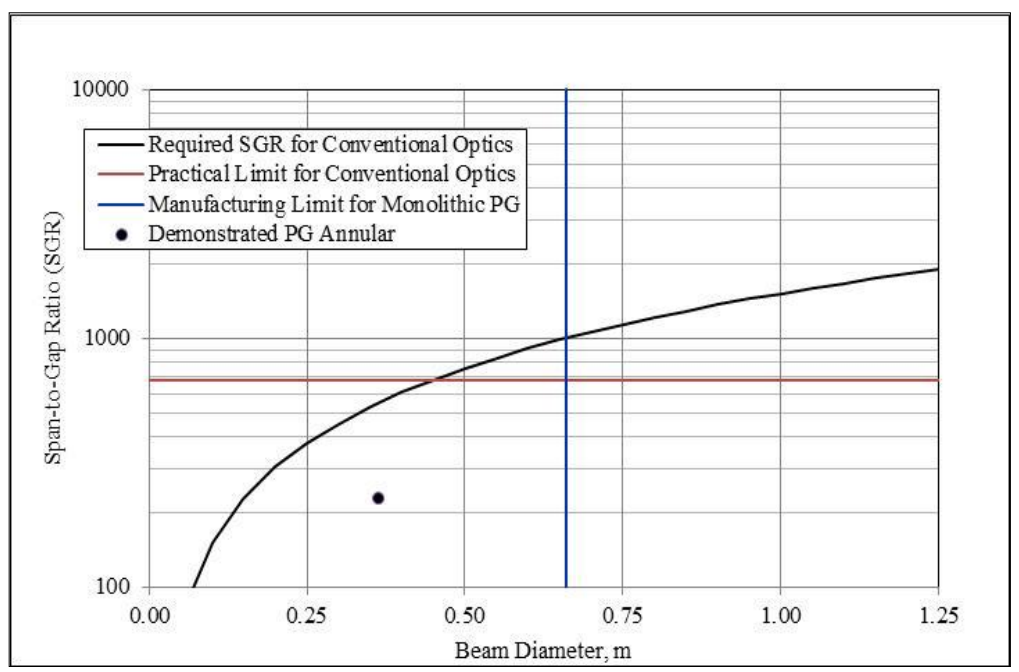

Figure 11. Span-to-Gap Ratio (SGR) vs. Beam Diameter for Conventional Ion Optics

input power capability of $30.6 \mathrm{~kW}$, at 4670 seconds $I_{s p}$. Nesting a NEXT thruster within the interior of this AGIEngine would yield a peak power of the hybrid of about $44 \mathrm{~kW}$. Figure 12 plots the peak power versus $I_{s p}$ for this NEXT/65 cm AGI-Engine Hybrid, scaled directly from maximum power vs. $I_{s p}$ demonstrated for the NEXT thruster. ${ }^{8}$ As indicated the input power for this hybrid ranges from about $15.3 \mathrm{~kW}$ to $44.2 \mathrm{~kW}$ over an $I_{s p}$ range of 2730 seconds to 4670 seconds.

Figure 13 plots peak power versus AGI-Engine beam diameter (outer diameter of the annulus), assuming the same peak power density as demonstrated with the NEXT thruster. For AGI-Engine beam diameters ranging from $0.45 \mathrm{~m}$ (somewhat larger than SOA NEXT thruster) to $0.65 \mathrm{~m}$ (nominal maximum for monolithic PG panels) the peak input power ranges from about $18.5 \mathrm{~kW}$ to 30.6 $\mathrm{kW}$. A maximum span of $14.4 \mathrm{~cm}$ and a SGR of $\sim 227$ were assumed. Also plotted is the maximum power point demonstrated with the NEXT thruster. Because of the increased anode surface area associated with the AGI-Engine concept there is the potential that even higher power and thrust densities than those demonstrated with conventional ion thrusters and shown on Figs. 12 and 13

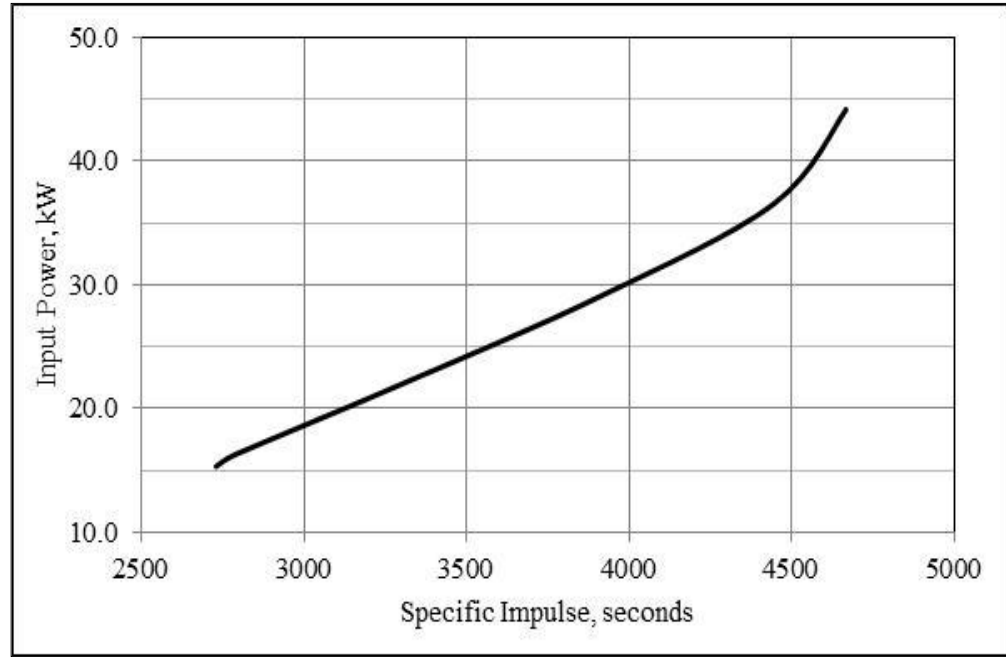

Figure 12. Input Power vs. Specific Impulse for NEXT/65 cm AGI-Engine Hybrid; Power Density Same as Demonstrated Previously with NEXT Thruster would be feasible. This will be discussed in the following section.

One means of circumventing the present manufacturing limitation of $\sim 66 \mathrm{~cm}$ diameter for individual PG panels would be to implement 'segmented' or 'unitized' electrodes to limit the required size of individual panels. The individual panels would then be clustered together and mechanically secured to a framework on the discharge chamber to create an annulus, with the annular construct maintained for increased thrust density and improved packaging. With a common span for all AGI-Engines one might employ a relatively-small 'optics-unit' that can be used (replicated) on each engine, with the quantity of the units required defined by the area of the annular channel. By having a common optics-unit, which might contain its own mounting assembly, one could reduce manufacturing 
costs and drive toward testing at the unit-level for qualifications. This "optics-unit" could be a circular set of optics, or it could be rectangular, or pie-wedge, etc. Examples of this multi-panel construction technique are illustrated in Figure 14. So long as this method is applied in a manner to achieve a high physical transparency at the plane of the screen electrode (a high 'packing-density') then this technique should not negatively impact discharge performance.

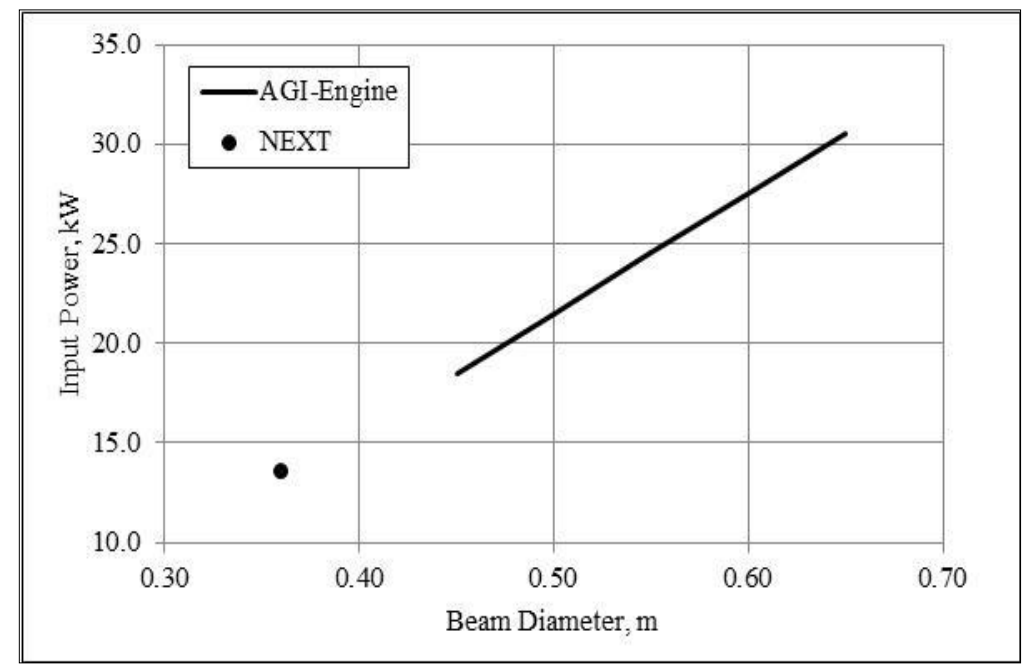

Figure 13. Peak Input Power vs. Beam Diameter (Outer Dia. Of Annulus) for AGI-Engine; Power Density Same as Demonstrated Previously with NEXT Thruster

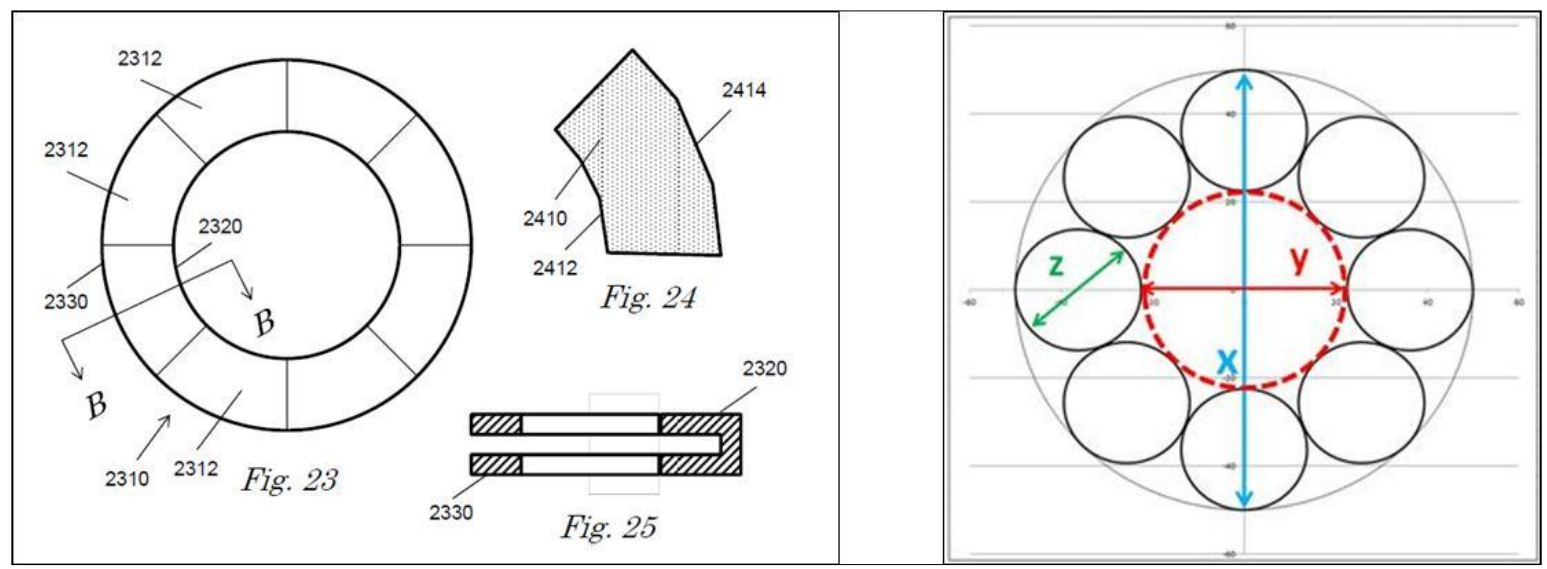

Figure 14. Examples of Multi-Panel Construction of PG Electrodes Arranged in an Annular Geometry: Segmented (Pie-Wedge) Electrodes, Left; Unitized (Circular) Electrodes, Right

By applying this multi-panel construction technique the maximum size of the AGI-Engine which could be built is only limited by considerations associated with spacecraft integration -1.5 meters in diameter or greater may be practical. Combining this construction technique with the MAGI-Engine concept (concentric ion rings) one could achieve very high power levels. As an example a MAGI-Engine consisting of 4 rings with a constant span of $17 \mathrm{~cm}$ and outer-most of $1.5 \mathrm{~m}$ diameter one could potentially achieve input power levels of $0.11 \mathrm{MW}$ at about 2000 seconds $I_{s p}$, and $>0.36 \mathrm{MW}$ processed at about 4500 seconds $I_{s p}$; same power density again as previously demonstrated for the NEXT thruster. These estimates are shown in Figure 15, a plot of input power vs. $I_{s p}$, for the 4ring MAGI-Engine along with the estimates from Fig. 12 of the NEXT/65 cm AGI-Engine hybrid. 
3. Maximum Power and Thrust Densities

There is evidence that ion thrusters are capable of operating at significantly higher power and thrust densities than is typically employed. Figure 16 is a plot of demonstrated thrust density vs. power density for a number of SOA xenon ion thrusters; NEXT, NSTAR, and XIPS. ${ }^{11}$ The data for all these thrusters are identified in the figure as 'Ring-Cusp', which specifies a particular magnetic circuit configuration $^{12}$ which is a common design feature of these thrusters. These thrusters also employ similar (2- or 3grid) high perveance ion optics electrode designs and operate in somewhatoverlapping specific impulse ranges (with NEXT running over the largest and inclusive range of 1410-4190 seconds). All data are at $\leq 4,300$ seconds $I_{s p}$.

The NSTAR thruster data of Fig. 16 includes its maximum power point and a previously-unpublished higher density point. The XIPS data is its standard high power throttle condition. The maximum point on the 'Ring-Cusp' cluster of data is from the NEXT thruster about $4.6 \mathrm{~N} / \mathrm{m}^{2}$ and $134 \mathrm{~kW} / \mathrm{m}^{2}$ thrust and power densities respectively corresponding to $13.60 \mathrm{~kW} P_{\text {in-t }}$ and 0.466 $\mathrm{N}$ thrust $(F)$. This represents the highest densities published for SOA xenon ion thrusters. The standard maximum power point for the NEXT thruster throttle table is also included.

The question arises what is practically inhibiting the NEXT thruster from operating at even higher densities. At the peak densities in Fig. 16 for the RingCusp data, the NEXT thruster is operating a beam current of 7.0 Amperes at $1800 \mathrm{~V}$ $V_{b p s}$, and total accelerating voltage of 2010 V. There is sufficient thermal margin to operate at much higher beam currents. Additionally, NEXT ion optics perveance at $2010 \mathrm{~V}$ total voltage should support a beam current of at least 15 Amperes. ${ }^{8}$ At the peak condition in Fig. 16 the thruster is operating at about 32 Amperes discharge (anode) current. What is experimentally observed above this value of anode current is the discharge becomes highly resistive and unstable, indicating that there is insufficient anode surface area to support higher currents. That is, the maximum thrust and power densities are limited by the source production - the ability to produce additional ion current - and not the ability of the optics to extract additional ion current. The effective anode surface area in a ring-cusp magnetic circuit discharge - the area which actively collects electron current from the plasma and sustains the discharge - is actually quite small relative to the total geometric surface area of anodepotential surfaces.

Supporting evidence for anode area limiting the source production comes from data obtained from a 'divergent field' thruster, also plotted on Figure 16. This thruster, with a $28.7 \mathrm{~cm}$ beam diameter was operated at $17.07 \mathrm{~kW} P_{i n}$ ${ }_{t}$ producing a thrust $0.577 \mathrm{~N}$; corresponding to a power density of $\sim 264 \mathrm{~kW} / \mathrm{m}^{2}$ and thrust density of $\sim 8.9 \mathrm{~N} / \mathrm{m}^{2}$. ${ }^{2}$ These values are believed to be the highest demonstrated for electrostatic ion thrusters. These values not only represent higher power and thrust densities than demonstrated for SOA thrusters, but are significantly higher in

12

American Institute of Aeronautics and Astronautics 
absolute power and thrust as compared to SOA ion thrusters, including the much larger NEXT ion thruster. This divergent field thruster employed a nearly-identical electrostatic ion optics design as compared to that of the NEXT and NSTAR thrusters. What is markedly different is the magnetic circuit employed.

The divergent field thruster, as the name implies, uses a weakly-divergent field to contain the discharge plasma and which is much less effective in containing primary electrons. In this design the effective anode area available for electron collection is believed to be equal to the total geometric surface area of anode-potential surfaces which even for this small diameter thruster is much larger than the effective anode surface area of the Ring-Cusp thrusters. So while the innovation of the ring-cusp magnet circuit design has brought about significant improvements (namely, high electrical efficiency, uniform plasma production, reduced electrode voltages and dramatically enhanced life) it may have done so at the expense of source production.

Given the similar ion optics designs of the thrusters plotted in Figure 16 they all operate at equivalent maximum beam voltages. One may therefore re-plot the data of Fig. 16 to illustrate the source production limit versus ion extraction limit consideration. Figure 17 is a plot of beam current density vs. thrust density for the same data of Fig. 16. As indicated, although all the ion optics electrostatic designs are comparable, the divergent field ion thruster operated at $\sim 2 \mathrm{X}$ the current density as compared to the maximum demonstrated for the RingCusp designs; about $137 \mathrm{~A} / \mathrm{m}^{2}$ vs. 69 $\mathrm{A} / \mathrm{m}^{2}$ at a thrust density of about $8.9 \mathrm{~N} / \mathrm{m}^{2}$ vs. $4.6 \mathrm{~N} / \mathrm{m}^{2}$.

One can estimate the current extraction limit of the electrostatic ion optics design employed by the thrusters in

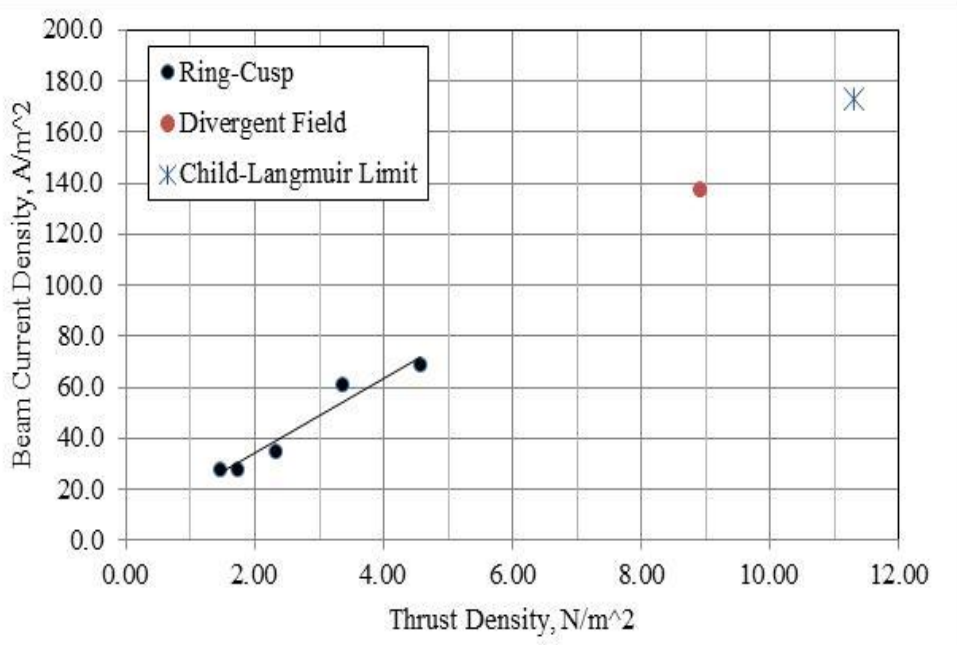

Figure 17. Ion Thruster Demonstrated Beam Current Density vs. Thrust Density; Child-Langmuir Limit for this Optics Design also plotted

Figs. 16 and 17 from a one-dimensional solution of Poisson's equation. This 'Child-Langmuir limit' (also plotted on Fig. 17) represents the maximum theoretical limit for this specific electrostatics design, and is estimated to be about $173 \mathrm{~A} / \mathrm{m}^{2}$ corresponding to a power density of about $336 \mathrm{~kW} / \mathrm{m}^{2}$ and a thrust density of $11.3 \mathrm{~N} / \mathrm{m}^{2}$. The maximum current density demonstrated by the Ring-Cusp thrusters is only $40 \%$ of this limit, whereas the divergent field thruster operated near $80 \%$ of the optics limit; the factor of 2 difference in capability is presumed to be the difference in anode area to support higher plasma densities (ion production and extraction). The calculated ChildLangmuir limited power density and thrust density, and those values demonstrated for the divergent field ion thruster, in fact comparable favorably to SOA high-power Hall thrusters. ${ }^{14}$

One of the many potential advantages of the AGI-Engine is the annular-shaped discharge chamber, which increases the effective anode-surface area for electron-collection as compared to a conventional cylindrically-shaped ion thruster of equivalent beam area. This increase in anode surface area should allow the AGI-Engine to operate at higher discharge currents and therefore potentially higher beam currents and input power levels than a SOA ion thruster of equivalent beam area for a given specific impulse. That is, the AGI-Engine should operate more closely to the Child-Langmuir limit (while retaining the advantages of the ring-cusp magnetic circuit) thereby utilizing more efficiently the perveance capability of the ion optics (source extraction limit), and not have a maximum input power level limited by the available anode surface area (source production limit).

If one assumes AGI-Engine operation at $80 \%$ of the Child-Langmuir limit - equivalent to that demonstrated with the divergent-field thruster, then the peak power vs. specific impulse for the NEXT/65 cm AGI-Engine hybrid, and peak input power vs. beam (outer annulus) diameter for the AGI-Engine concept can be re-plotted for Figs. 12 and 13, and these are shown in Figures 18 and 19. In Figure 18 the estimated input power vs. $I_{s p}$ is plotted for the NEXT/65 cm AGI-Engine hybrid for two conditions: operating at the same power density as previously demonstrated with NEXT; and enhanced power density operating at $80 \%$ of the Child-Langmuir limit. The latter yields $\sim 2 \mathrm{X}$ increase in $P_{\text {in-t }}$, ranging from about $30.9 \mathrm{~kW}$ at 2730 seconds $I_{s p}$ to about $89 \mathrm{~kW}$ at 4670 seconds $I_{s p}$. Similarly Figure 19 plots the peak input power vs. beam diameter (over 0.45-0.65 m range annulus outer diameter) for the AGI-Engine concept at two different power densities: both NEXT demonstrated; and enhanced power density. A maximum span of $14.4 \mathrm{~cm}$ and a SGR of $\sim 227$ were assumed. With a factor of $\sim 2 \mathrm{X}$ increase due to 
larger anode surface area there is the potential for the AGI-Engine concept to operate at peak input powers of $37 \mathrm{~kW}$ to $62 \mathrm{~kW}$ for these sizes; operating at $80 \%$ of the ion optics Child-Langmuir Limit.

\section{B. Mission Applications}

The practical implementation of the AGI-Engine and MAGI-Engine concepts should be relatively straightforward, as it represents a natural extension in mission capabilities and applications engendered by ion propulsion. Less clear are the implementation issues associated with the alternative configurations of the concept; in particular the DMH-Engine, with potential hybridization of the annular ion rings with an HET, or other devices.

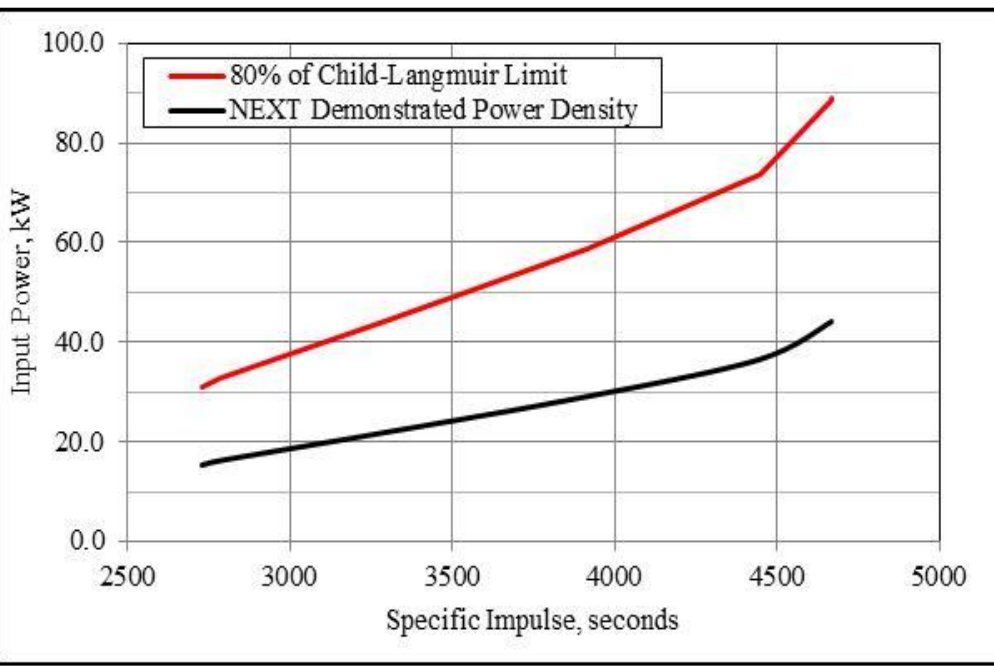

For the DMH-Engine a development and implementation assessment is required. In the instance of an ion/HET hybrid, a bimodal engine capability may be interest. As an example, many mission concepts have periods of "urgency" in which it is desirable to effect orbit change as quickly as possible. These urgent periods are often matched with periods of less urgency where other concerns like fuel efficiency may be more important. Many examples exist for both round trip mission architectures and more conventional mission designs. A DMHEngine may allow a vehicle to throttle up to high thrust settings during urgent periods and throttle down to low settings when appropriate. Other concepts could employ this urgent/non-urgent concept of operations, including most round trip architectures where one mission leg is considered urgent (delivery of a payload, orbit change to conduct time-sensitive observation, etc.) and the return leg is not as urgent. Designs using SOA EP systems do not have the same flexibility and other aspects of the mission must be sacrificed as a result.

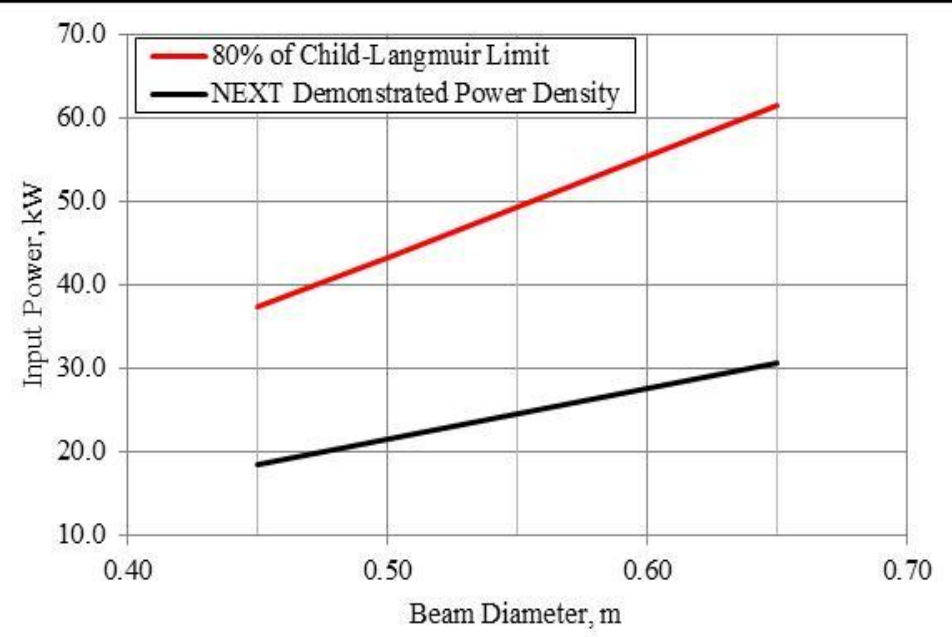

A common flaw with developing a single-solution is that the system is often

Figure 19. Peak Input Power vs. Beam Diameter (Outer Dia. Of Annulus) for AGI-Engine; Two Different Power Densities Assumed overdesigned or cumbersome to implement as compared to a system optimized for the single application. In the case of the DMH-Engine one must consider the implications of the engine architecture on elements such as the power processing unit. Compatibility and commonality of the circuit topology to operate both the HET component discharge and ion component beam must be accounted for. A modular power system approach would also be desired to simply add modules as the power requirements increase.

\section{Full-Scale High-Power Annular Engine Development}

Under a phase 2 effort the objective is to demonstrate scalability of the AGI-Engine concept to high power via the design, fabrication, and test of a full-scale Annular Engine Demonstrator, executing the tasks indicated in Table 2. This represents a natural progression from that which was achieved to date in the first phase; proof-of-concept demonstration of the sub-scale Annular Engine. This section discusses some design considerations and a pointdesign for a second-generation high-power AGI-Engine, and associated implementation tasks. 


\section{A. Design Considerations}

To meet the objectives identified in Table 2 for the full-scale Engine it needs to be sufficiently large to have the potential to operate in the $\sim 10$ 's of $\mathrm{kW}$ power range. This sizing will also establish whether or not the azimuthal and radial discharge and beam uniformity demonstrated with the sub-scale Engine $^{5}$ can be maintained using a singlecathode design.

The larger size will also provide the opportunity to address manufacturing, assembly, and test issues associated with larger-area PG electrodes. The second-generation Engine diameter will be purposely limited to $\leq 66 \mathrm{~cm}$ annulus outside diameter so that a single (monolithic) panel construction technique can be employed for the ion optics.

The Engine must also be scaled appropriately to ensure that there is sufficient anode surface to enable operation closer to the Child-Langmuir limit than is the case for conventional ion thrusters while maintaining relatively-low discharge losses. With this scaling comes the opportunity to assess whether or not higher discharge currents and higher beam current densities can be supported. The interior diameter of the annulus will be sufficient large to accommodate either a centrally-mounted neutralizer cathode assembly, or integration of a centrally-mounted HET to investigate a DMH-Engine configuration. Consideration is being given to investigating alternative magnet circuit designs than are employed in SOA ion thrusters.

\section{B. Design}

The full-scale AGI-Engine is being designed to accommodate a maximum beam diameter of $65 \mathrm{~cm}$. It will utilize a single hollow cathode installed at a radial position corresponding to the center of the annulus. The ion optics electrodes will have comparable electrostatic design to that employed with the sub-scale Engine PG electrodes, with span in the range of 8.5-19.5 cm corresponding to an SGR range of $~ 130-300$.

One possible magnetic circuit for the Engine, a ring-cusp design, is shown in Figure 20, a cross-section of the discharge chamber. The magnetic circuit was designed using a commercial finite element, magnetostatic solver. The solver evaluates the magnetostatic field equations using a Newton-Rhapson iteration methodology. Convergence is determined by energy error, which is evaluated on each iteration pass. This magnet circuit design features 15 magnet rings. One of the 15 rings intersects the cathode position and is thus split at that location. The magnetic geometry has a closed $50 \mathrm{G}$ contour line and features a large, field-free region, which should correspond to an equally large, uniform plasma core once the discharge is initiated. The $30 \mathrm{G}$ line is shown in the figure as the outermost field contour. Even this contour is nearly closed as well. The magnetic field gradient on centerline of the discharge chamber at the expected

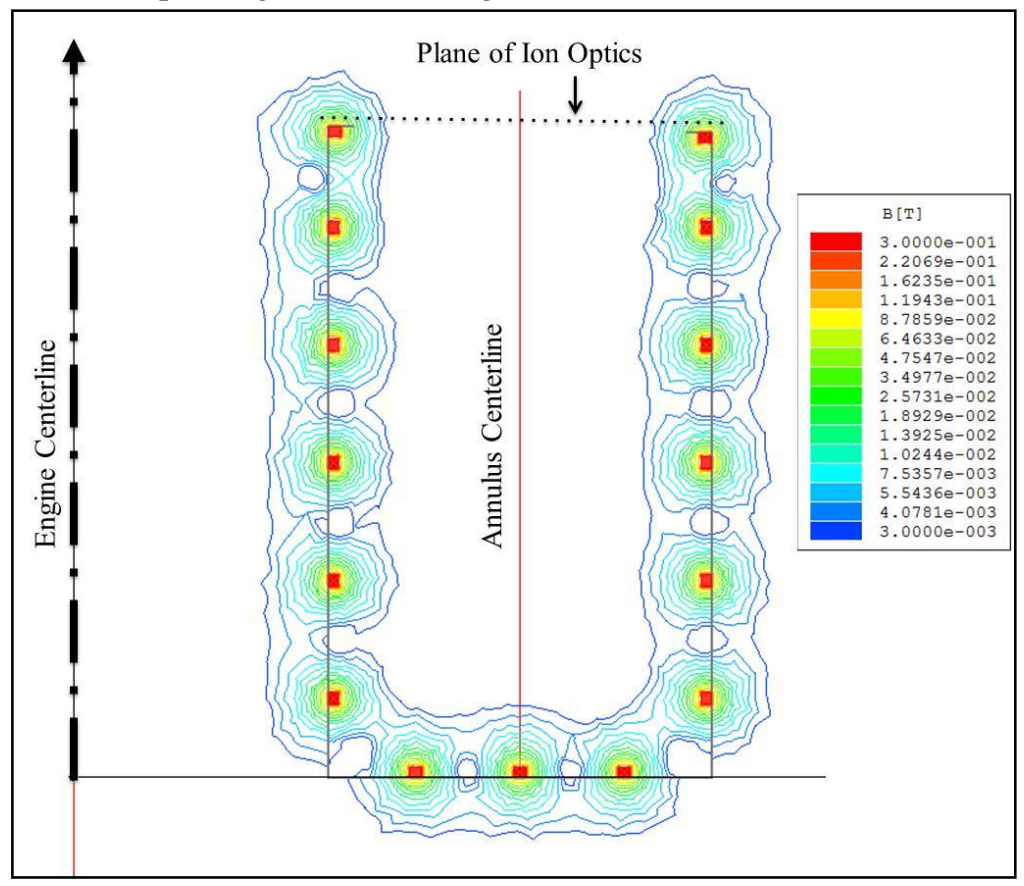

Figure 20. Ring-Cusp Magnet Circuit for $65 \mathrm{~cm}$ AGI-Engine

location of the cathode tip and at points further downstream decreases with distance. This profile is a prerequisite for stable operation. Despite the fact that the rings are located on cylindrical surfaces of different radii, the contour profile is largely symmetrical. The magnetic circuit design preserves the layout heritage of the sub-scale Engine. Preservation of this magnet layout should insure that gradients in the magnetic field are similar. It has been conjectured that such gradients drive azimuthal electron flow, leading to the observed high uniformity of the discharge plasma.

Magnetic cusp structure at each magnet ring is also highly developed; that is, with the exception of the pole piece magnet ring, the layout of the magnetic rings is such that the magnetic loss cones are directed toward the discharge plasmas - essentially allowing plasma flow from the bulk plasma into the cusp. This is to be contrasted with those geometries where the magnetic flux spatial profile at a given ring is distorted to the point that the ring is 
essentially magnetically isolated from the discharge plasma. In such a case, plasma diffusion to such a ring would be greatly reduced. Such a condition would reduce the maximum current carrying capacity of the engine. The sheer number of magnet rings, 15, greatly increases the magnetic cusp loss area thereby greatly increasing the engine's discharge current handling capacity. The capacity to process the larger discharge currents means that the ion production rate and thus beam current upper limit is greatly increased over conventional engines of similar diameter.

Figure 21 plots the projected input power vs. $I_{s p}$ for the second-generation AGI-Engine. The Engine is assumed to have an outside beam diameter of $65 \mathrm{~cm}$, with $14.4 \mathrm{~cm}$ span and SGR of 227. This Engine would have more than $2 X$ the beam area and nearly $4 \mathrm{X}$ the anode area of the NEXT thruster. In Fig. 21 two curves are displayed: one assuming operation at the NEXT thruster maximum demonstrated power density ( $40 \%$ of the Child-Langmuir Limit) ranging from about $10.6 \mathrm{~kW}$ to $30.6 \mathrm{~kW}$; and a second curve at $80 \%$ of the Child-Langmuir limit ranging from about $21.4 \mathrm{~kW}$ to $61.5 \mathrm{~kW}$. This corresponds to about $0.5-1.0 \mathrm{~N}$ thrust for the lower curve, and 1.0-2.1 $\mathrm{N}$ for the upper curve. As indicated the specific impulse ranges from about 2730 seconds to 4670 seconds.

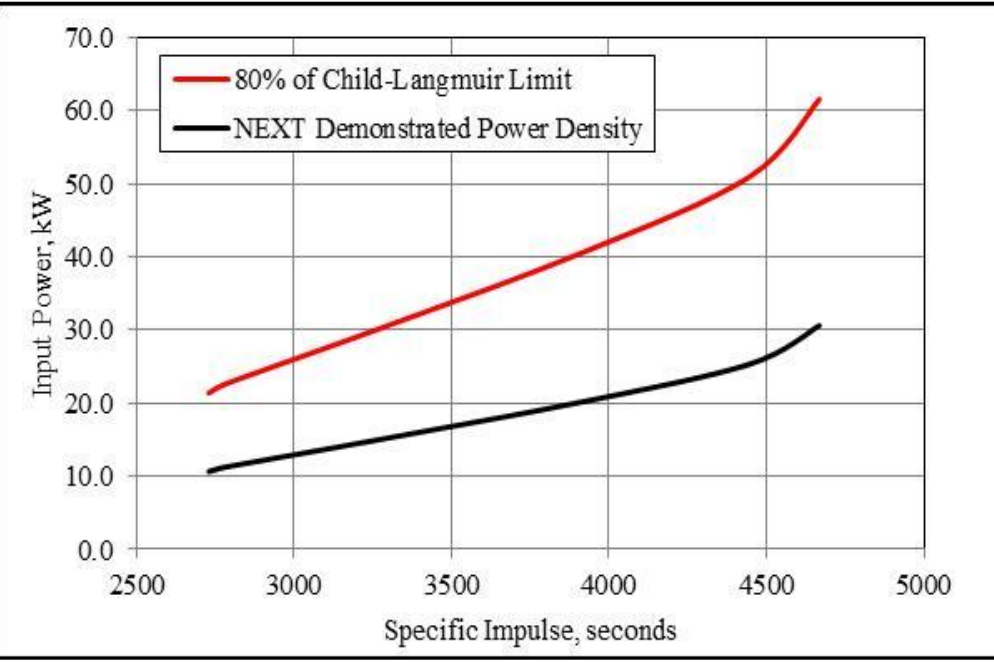

Figure 21. Input Power vs. Specific Impulse for $65 \mathrm{~cm}$ AGIEngine; Two Different Power Densities Assumed

\section{Implementation Tasks}

The development phases for the second-generation AGI-Engine will closely follow the process applied for the breadboard Engine development. Initial testing will be to assess discharge performance. The testing will characterize the discharge with internal plasma diagnostics including Mach probes, and fast frame rate cameras to track instabilities. These tests will be conducted to verify stable annular discharge operation, determine the axial and radial uniformity of the annular plasma with estimates of beam current density and beam flatness, and quantify discharge electrical efficiency. These tests will be performed under 'simulated beam extraction' up to discharge power conditions equivalent to at least $30 \mathrm{~kW}$ Engine input power. This methodology is often employed to characterize discharge operation in an efficient and cost-effective manner. ${ }^{15}$ It reduces test facility requirements, and defers the need to commit the large resources required to build large-area ion optics until such time the discharge concept has been validated. This technique was successfully employed in Phase 1, with very-precise correlation between the estimated breadboard Engine performance with simulated beam extraction and that obtained subsequently with beam extraction. ${ }^{3}$

Separately an optics scaling assessment for the full-scale Demonstrator Engine will be performed. This involves two tasks. One task will be to conclude the electrostatic characterization of sub-scale PG flat-panel annular electrodes operating on the sub-scale breadboard AGI-Engine. This will be to define the perveance per-unit-area as a function of beam current density, and obtain additional plume measurements. A task will be conducted 'on-thebench' to document mechanical deflection of the sub-scale PG flat annular electrodes under electrostatic load as a function of applied voltage, electrode thickness, and unsupported span. These data will feed into a model to predict the mechanical behavior of larger diameter annular optics. Both activities will provide data for the design and manufacturing of the large-area (65-cm dia.) annular optics for the Full-Scale Annular Engine Demonstrator.

\section{Conclusion}

This publication documented the development status of the Annular Geometry Ion-Engine, which is the design core of the Next-Generation Electric Propulsion Thruster concept. The AGI-Engine itself represents a potential pathway to dramatically increasing the power and thrust densities of ion thrusters.

Progress was demonstrated in the maturation of the AGI-Engine with the design, fabrication, and testing of subscale ( $\sim 40 \mathrm{~cm}$ dia.) flat annular geometry ion optics electrodes manufactured from pyrolytic graphite (PG). The 
electrodes are of high-perveance design and were integrated with the annular discharge chamber and successfully operated at electric fields up to about $2200 \mathrm{~V} / \mathrm{mm}$ at multiple throttle levels of the NEXT thruster during which these tests demonstrated very-high beam collimation and high beam flatness. The annular optics have a span-to-gap ratio of less than $1 / 2$ that of the NEXT ion optics while having a beam area 97\% of the NEXT beam area.

Scalability and scaling relations for the AGI-Engine concept were discussed. Increases in both unsupported span and in electric field strength for flat PG electrodes beyond that demonstrated with the sub-scale flat PG electrodes are likely feasible. However there are manufacturing limitations to the fabrication size of individual PG panel sizes to about $66 \mathrm{~cm}$ diameters. Even with this constraint this still represents a substantial increase in beam area, as compared to that demonstrated with conventional ion thrusters. For example a $65 \mathrm{~cm}$ beam diameter AGI-Engine with a span of $14.4 \mathrm{~cm}$ would yield a beam area of approximately $~ 2.3 \mathrm{X}$ that of the NEXT thruster beam area. If this $65 \mathrm{~cm}$ AGI-Engine were then simply operated at the same peak power density as that previously demonstrated with the NEXT thruster it would have an input power capability ranging from about $10.6 \mathrm{~kW}$ to $30.6 \mathrm{~kW}$ over a specific impulse range from about 2730 seconds to 4670 seconds.

One means of circumventing the present manufacturing limitation for individual PG panels would be to implement 'segmented' or 'unitized' electrodes to limit the required size of individual panels. By applying this multi-panel construction technique the maximum size of the AGI-Engine which could be built is only limited by considerations associated with spacecraft integration - 1.5 meters in diameter or greater may be practical - yielding Engines with multi-hundred $\mathrm{kW}$ power handling capability at $<5000$ seconds $I_{s p}$.

Maximum power and thrust densities for ion thrusters were discussed. There is evidence that ion thrusters are capable of operating at significantly higher power and thrust densities than is typically employed with today's SOA devices. Ion thrusters were operated at $\sim 9 \mathrm{~N} / \mathrm{m}^{2}$ and $\sim 264 \mathrm{~kW} / \mathrm{m}^{2}$ more than two decades ago, at nearly $80 \%$ of the estimated Child-Langmuir limit of the ion optics. This is $\sim$ twice the thrust and power density of the NEXT ion thruster and more than $2 \mathrm{X}$ for other SOA ion thrusters. This enhanced capability is likely associated with larger effective anode surface areas, which is also an attribute of the AGI-Engine design concept. The larger anode surface area of the AGI-Engine should permit operation at higher power and thrust densities than SOA thrusters.

Full-scale AGI-Engine development is underway to demonstrate scalability of the annular discharge and annular ion optics concept. A $65 \mathrm{~cm}$ AGI-Engine, using the same electrostatic design of SOA ion optics could potential operate at $\geq 2 \mathrm{X}$ the power and thrust densities of the NEXT ion thruster. This would yield a peak input power ranging from about $21 \mathrm{~kW}$ to $62 \mathrm{~kW}$ over the $2730-4670$ seconds $I_{s p}$ range.

\section{Acknowledgments}

The support of Mr. George Soulas, Mr. Jesus Lopez, and Mr. John Veneziano of NASA GRC for design assistance and manufacturing of the pyrolytic graphite ion optics and Mr. Kevin McCormick of NASA GRC for thruster design assistance and assembly is gratefully acknowledged. This work was supported under the GRC Center Innovation Fund program sponsored by NASA's Office of Chief Technologist, and The Aerospace Corporation's Independent Research and Development Program.

\section{References}

${ }^{1}$ Williams, G.J., et al., "Results of the 2000 hr Wear Test of the HiPEP Ion Thruster with Pyrolytic Graphite Ion Optics," AIAA Paper No. 2006-4668, 42 ${ }^{\text {nd }}$ Joint Propulsion Conference, July, 2006

${ }^{2}$ Patterson, M.J., Pinero, L., and Sovey, J.S., "Near-Term High Power Ion Propulsion Options for Earth-Orbital Applications," AIAA Paper No. 2009-4819, 45 ${ }^{\text {th }}$ Joint Propulsion Conference, August, 2009

${ }^{3}$ Patterson, M.J., Herman, D., Shastry, R., Van Noord, J., Foster, J.E., "Annular-Geometry Ion Engine: Concept, Development Status, and Preliminary Performance," AIAA Paper No. 2012-3798, 48 " Joint Propulsion Conference, July, 2012

${ }^{4}$ Patterson, M.J., "Next-Generation Electric Propulsion Thrusters," AIAA Paper No. 2011-4812, 47 $7^{\text {th }}$ Joint Propulsion Conference, July, 2011

${ }^{5}$ Shastry, R., Patterson, M.J., Herman, D., Foster, J.E., "Current Density Measurements of an Annular-Geometry Ion Engine," AIAA Paper No. 2012-4186, $48^{\text {th }}$ Joint Propulsion Conference, July, 2012

${ }^{6}$ Haag, T.W., and Soulas, G., "Performance of 8-cm Pyrolytic Graphite Ion Thruster Optics," AIAA Paper 2002-4335, 38 ${ }^{\text {th }}$ Joint Propulsion Conference, July, 2002

${ }^{7}$ Haag, T.W., and Soulas, G., "Performance and Vibration of 30-cm Pyrolytic Ion Thruster Optics," AIAA Paper 2003-4557, 39th Joint Propulsion Conference, July, 2003

${ }^{8}$ Patterson, M.J., "NEXT Study of Thruster Extended Performance (NEXT STEP)," AIAA Paper No. 2006-4664, 42nd Joint Propulsion Conference, July, 2006 
${ }^{9}$ Pollard, J.E, Diamant, K.D., Crofton, M.W., Patterson, M.J., and Soulas, G.C., "Spatially-Resolved Beam Current and Charge-State Distributions for the NEXT Ion Engine" AIAA Paper No. 2010-6779, 46 ${ }^{\text {th }}$ Joint Propulsion Conference, July, 2010

${ }^{10}$ Kaufman, H.R., "Technology of Electron-Bombardment Ion Thrusters," Advances in Electronics and Electron Physics, Vol. 36, Academic Press, Inc., New York 1974, pp. 265-373

${ }^{11}$ W.G. Tighe, et al., "Performance Evaluation of the XIPS 25-cm Thruster for Application to NASA Discovery Missions," AIAA Paper No. 2006-4666, 42nd Joint Propulsion Conference, July, 2006

${ }^{12}$ J. Sovey, J.S., "Improved ion containment using a ring-cusp ion thruster," Journal of Spacecraft and Rockets, Vol. 21, No. 5 (1984), pp. 488-495

${ }^{13}$ Patterson, M.J. and Rawlin, V.K., "Performance of 10-kW Class Xenon Ion Thrusters," AIAA Paper No. 88-2914, 24 Joint Propulsion Conference, July, 1988

${ }^{14}$ Soulas, G.C., et al., "Performance Test Results of the NASA-457M v2 Hall Thruster," AIAA Paper No. 2012-3940, 48 Joint Propulsion Conference, July, 2012

${ }^{15}$ Brophy, J.R., "Simulated Ion Thruster Operation Without Beam Extraction," AIAA Paper No. 90-2655, 26th Joint Propulsion Conference, July, 1990 